\title{
Effect of Delayed Cord Clamping on Iron Stores in Infancy
}

\author{
SIDDARTH RAMJI \\ Director-Professor, Department of Neonatology, Maulana Azad Medical College, New Delhi, India. \\ siddarthramji@gmail.com
}

$\mathrm{T}$ his issue of Indian Pediatrics has published a meta-analysis on the effect of delayed cord clamping in term infants on anemia in infancy by Fu, et al. [1]. The present systematic review included 12 studies which had 993 infants in the delayed cord clamping (DCC) group and 989 infants in early cord clamping group (ECC). The review concluded that the hemoglobin and serum ferritin were significantly higher in infancy in the DCC group, compared to the ECC group. The conclusions are not different from the review by McDonald, et al. [2], which included 5 studies (620 in DCC and 532 in ECC), and concluded that infants in the late clamping group were less likely to be anemic at 3-6 months of age. However, both reviews observed high heterogeneity in the reported results, which could be due several reasons - the way iron deficiency is defined, maternal iron status, type of feeding, proportion of low birth weight babies, etc. The review by Fu, et al. [1] has added two other possible variables that could potentially contribute to the heterogeneity i.e, ethnicity and timing of delayed cord clamping. Studies that use serum ferritin alone as a measure of iron deficiency, without concomitant hemoglobin measurement, are fraught with the risk of missing iron deficiency if serum ferritin is elevated due to infection (as an inflammatory response). This could be a potential confounding, especially in lowresource settings where infection rates tend to be high. Studies observing the effect of interventions on anemia in infancy should therefore include C-reactive protein to exclude concomitant infection, along with serum ferritin and hemoglobin measurements.

While there is evidence of the benefits of DCC on iron stores in term infants, its effect on iron stores in preterm infants beyond the neonatal period is limited. In a small trial in 37 preterm neonates over a decade ago, Ultee, et al. [3] observed that at 10 weeks, the DCC group had higher hemoglobin values. Trials of DCC versus ECC in preterm infants may not be easy in the present time, as clamping the cord late is increasingly being recommended and practiced as the norm, and thus could pose an ethical challenge for the conduct of such trials. The only possibility could be varying the timing of cord clamping beyond 60 seconds. The appropriate timing of cord clamping when benefits could cease to occur or harm (if any) could manifest, is unknown and hence should be explored. One of the reasons to examine such questions could be the need to validate observations such as by $\mathrm{Fu}$, et al. [1] that benefits of increased iron stores may not accrue if cord is clamped beyond 120 secs.

There have been reports that low cord ferritin could be a potential biomarker to predict brain iron deficiency and dysfunction as evidenced by lower psychomotor scores at 1 year with iron deficiency due to maternal gestational diabetes [4]. Gupta and Ramji [5], in a randomized trial, observed that term infants born to anemic mothers who underwent DCC were at lower odds for anemia at 3 months compared to those in the ECC group. Thus, in regions of the world with high burden of maternal anemia, DCC has the potential to positively impact iron stores in infants and their neurodevelopment outcomes as has been demonstrated by Andersson, et al. [6] in term infants in Sweden. To see its effect in low-resource settings, the same authors had designed a trial comparing DCC and ECC on neurodevelopment to be conducted in Nepal [7]; the trial was registered in 2014 but is apparently not recruiting as per information available at the trial registry site.

While trialists continue in their endeavor to untangle the influence of the effects of confounding on the timing of cord clamping and iron stores, current practice guidelines to delay cord clamping to atleast 60 seconds should be vigorously pursued given the available evidence of its benefits, which possibly goes beyond improving iron status in infancy and more importantly no harm having been demonstrated.

Competing interests: None stated; Funding: None.

\section{REFERENCES}

1. Fu X, Dang D, Li S, Xu Z, Wu H. Effect of delayed versus early cord clamping on improving anemia in term infants aged two months or older - A meta-analysis. Indian Pediatr. 
EDITORIAL

2020;57:815-9.

2. McDonald SJ, Middleton P, Dowswell T, Morris PS. Effect of timing of umbilical cord clamping of term infants on maternal and neonatal outcomes. Cochrane Database Syst Rev. 2013;2013:CD004074.

3. Ultee CA, van der Deure J, Swart J, Lasham C, van Baar AL. Delayed cord clamping in preterm infants delivered at 34-36 weeks' gestation: A randomized controlled trial. Arch Dis Child Fetal Neonatal Ed. 2008;93:F20-3.

4. Cusick SE, Georgieff MK, Rao R. Approaches for reducing the risk of early-life iron deficiency-induced brain dysfunction in children. Nutrients. 2018;10:227.
5. Gupta R, Ramji S. Effect of delayed cord clamping on iron stores in infants born to anemic mothers: A randomized controlled trial. Indian Pediatr. 2002;39:130-5.

6. Andersson O, Lindquist B, Lindgren M, Stjernqvist K, Domellöf M, Hellström-Westas L. Effect of delayed cord clamping on neurodevelopment at 4 years of age: A randomized clinical trial. JAMA Pediatr. 2015;169:631-8.

7. Kc A, Målqvist M, Rana N, Ranneberg LJ, Andersson O. Effect of timing of umbilical cord clamping on anaemia at 8 and 12 months and later neurodevelopment in late pre-term and term infants; A facility-based, randomized-controlled trial in Nepal. BMC Pediatr. 2016;16:35. 